TRANSACTIONS OF THE

AMERICAN MATHEMATICAL SOCIETY

Volume 356, Number 8 , Pages 3283-3300

S 0002-9947(03)03514-1

Article electronically published on December 12, 2003

\title{
INFINITELY MANY SOLUTIONS TO FOURTH ORDER SUPERLINEAR PERIODIC PROBLEMS
}

\author{
MONICA CONTI, SUSANNA TERRACINI, AND GIANMARIA VERZINI
}

\begin{abstract}
We present a new min-max approach to the search of multiple $T$-periodic solutions to a class of fourth order equations

$$
u^{i v}(t)-c u^{\prime \prime}(t)=f(t, u(t)), \quad t \in[0, T],
$$

where $f(t, u)$ is continuous, $T$-periodic in $t$ and satisfies a superlinearity assumption when $|u| \rightarrow \infty$. For every $n \in \mathbb{N}$, we prove the existence of a $T$-periodic solution having exactly $2 n$ zeroes in $(0, T]$.
\end{abstract}

\section{INTRODUCTION}

Let us consider the fourth order equation

$$
u^{i v}(t)-c u^{\prime \prime}(t)=f(t, u(t)), \quad t \in[0, T] .
$$

We seek $T$-periodic solutions having a prescribed number of simple zeroes on $(0, T]$ in the case when $c \geq-(\pi / T)^{2}, f: \mathbb{R} \times \mathbb{R} \rightarrow \mathbb{R}$ is continuous, $T$-periodic in $t$ and satisfies a superlinearity assumption at infinity:

$$
\lim _{|u| \rightarrow \infty} \frac{f(t, u)}{u}=+\infty
$$

Fourth order equations are the object of an intensive study and a great effort is performed in order to understand the structure of the set of the periodic motions. We mention the recent papers dealing with second order materials, with the extended Fisher-Kolmogorov equation, the Swift-Hohenberg equation and travelling waves in suspension bridges (see e.g. [10, 13, 15, 16, 17, 18, 20, 4] and the references therein). Nevertheless, to our knowledge, only a few papers (we recall 11, 11, 8, 19]) deal with nonlinearities having a behavior as in (2), that is, $f(t, u)$ is superlinear in $u$ and has the same sign as $u$ at infinity; moreover, only [1] 11] deal with the periodic boundary condition. Our contribution is the following:

Theorem 1.1. Let $c \geq-(\pi / T)^{2}, f: \mathbb{R}^{2} \rightarrow \mathbb{R}$ be a continuous function, $T$-periodic in the $t$ variable, and satisfying the following assumptions:

$\left(f_{1}\right)$ set $F(t, v)=\int_{0}^{v} f(t, x) d x$; there exists $\gamma>0$ such that

$$
f(t, v) v \geq(2+\gamma) F(t, v) \quad \forall v \in \mathbb{R}, t \in(0, T),
$$

Received by the editors May 25, 2001 and, in revised form, March 21, 2003.

2000 Mathematics Subject Classification. Primary 34B15; Secondary 58E05, 47J10.

Key words and phrases. Oscillating solutions, fourth order equations, boundary value problems, variational methods.

This research was supported by MURST project "Metodi Variazionali ed Equazioni Differenziali Non Lineari". 
$\left(f_{2}\right) f(t, v) v>0$ if $v \neq 0, t \in(0, T)$,

$\left(f_{3}\right) \lim _{v \rightarrow 0} \frac{f(t, v)}{v}=0$ uniformly in $t$.

Then, for every fixed integer $n \geq 1$, (1) has at least one $T$-periodic solution having precisely $2 n$ simple zeroes in $(0, T]$.

It is worth noticing that under the above assumptions there are no one-sign periodic solutions to (11). As an example of nonlinearity fulfilling all the assumptions of the theorem we can consider

$$
f(t, u)=(2+\cos t) u^{3},
$$

although we do require neither oddness nor other symmetry conditions.

The existence of solutions to boundary value problems associated to (1) has been proved, under rather strong restrictions on the nonlinearity $f$, by Afuwape, Mawhin and Zanolin [1. More recently, a continuation approach has been proposed by Mawhin and Zanolin [11], in order to prove the existence of at least one solution, in the special case $f(t, u)=g(u)+e(t)$, with a superlinear nonlinearity $g$. In the autonomous superlinear case, the fixed energy problem has been studied by Garcia in [8. The paper [19] deals with disconjugate operators combined with superlinear nonlinearity and includes our equation in the case $c=0$; the main goal there consists in the application of Rabinowitz's Global Bifurcation Theorem to the case of separated boundary conditions and cannot be extended to the periodic problem. Coming to our result, we wish to point out that our Theorem 1.1 provides not only existence but also multiplicity of solutions: in particular the existence of a $T$-periodic solution with $2 n$ simple zeroes is proved for each positive integer $n$.

In the present paper we shall introduce a new min-max procedure for the search of multiple solutions characterized by nodal properties. Although the proof shows relevant differences, the method was inspired by that introduced by two of the authors in 5, 6] when dealing with the second order case. The combination of variational methods and nodal properties was introduced in the literature by Nehari in [14. Since then, several variants of the original Nehari's method have been developed mainly for the case of second order equations (see e.g. [21, 2, 7]). We wish to stress that Nehari's method cannot be directly extended, by its nature, to the case of higher order equations.

We point out that our technique can also be applied to different boundary value problems associated to the equation. For instance, for the Dirichlet problem and under the same assumptions as in Theorem 1.1. we can prove the existence of one pair of solutions having $k$ simple zeroes, for all $k \geq 1$; this case, however, could also be covered by the bifurcation theory developed in [19].

\section{A VARiational PRinciple With tWo CONSTRAints}

This section is devoted an abstract min-max method that provides critical points for functionals in the presence of a two-components constraint. The final result of this section will be the key argument in proving Theorem 1.1 in Section 3

Our constraint will be defined by two inequalities

$$
\begin{aligned}
& G_{1}(u) \leq 1, \\
& G_{2}(u) \leq 1 .
\end{aligned}
$$


We seek critical points of a given functional $J$ satisfying the above inequalities. Of course, since we are interested in true critical points of $J$, we need to take into account the behavior of the gradients $\nabla J, \nabla G_{i}$ on the boundaries $G_{i}=1$. The simplest and most common case is when $\nabla J(u)$ and (say) $\nabla G_{1}(u)$ agree (in the sense that they share a common pseudo-gradient field) on the boundary $G_{1}(u)=1$. Then, any standard variational method will provide a critical point fulfilling the constraint. A more unusual case is when $\nabla J(u)$ and (say) $-\nabla G_{2}(u)$ agree in some sense on $G_{2}(u)=1$. However, this situation still gives the existence of a critical point inside the constraint, provided there is a nontrivial topology relative to the boundary $G_{2}(u)=1$. In the following we shall need to work with a combination of the two cases. We shall define a suitable min-max procedure that, together with a compatibility condition between $\nabla J, \nabla G_{1}, \nabla G_{2}$, will lead to the existence of a critical point fulfilling the constraint.

To start with, let $H$ be a Hilbert space, $J, G_{1}, G_{2} \in C^{1}(H, \mathbb{R})$ and let $a, b \in \mathbb{R}$, $a<b$. Let

$$
\Omega(a, b)=\left\{u \in H: J(u) \in[a, b], G_{1}(u) \leq \frac{3}{2}, G_{2}(u) \leq \frac{3}{2}\right\}
$$

and let us write the set $\Omega$ in four components as follows:

$$
\begin{aligned}
& \Omega_{1}(a, b)=\left\{u \in \Omega(a, b): G_{1}(u) \leq \frac{1}{2}, G_{2}(u) \leq \frac{1}{2}\right\}, \\
& \Omega_{2}(a, b)=\left\{u \in \Omega(a, b): G_{1}(u) \leq \frac{1}{2}, G_{2}(u) \geq \frac{1}{2}\right\}, \\
& \Omega_{3}(a, b)=\left\{u \in \Omega(a, b): G_{1}(u) \geq \frac{1}{2}, G_{2}(u) \leq \frac{1}{2}\right\}, \\
& \Omega_{4}(a, b)=\left\{u \in \Omega(a, b): G_{1}(u) \geq \frac{1}{2}, G_{2}(u) \geq \frac{1}{2}\right\} .
\end{aligned}
$$

Now we define

$$
\Lambda(a, b)=\bigcup_{i=1}^{4} \Lambda_{i}(a, b) \subset \Omega(a, b) \times \mathbb{R}^{-} \times \mathbb{R}^{-},
$$

where

$$
\begin{aligned}
& \Lambda_{1}(a, b)=\left\{\left(u, \lambda_{1}, \lambda_{2}\right): u \in \Omega_{1}(a, b), \lambda_{1}=0, \lambda_{2}=0\right\}, \\
& \Lambda_{2}(a, b)=\left\{\left(u, \lambda_{1}, \lambda_{2}\right): u \in \Omega_{2}(a, b), \lambda_{1}=0, \lambda_{2} \leq 0\right\}, \\
& \Lambda_{3}(a, b)=\left\{\left(u, \lambda_{1}, \lambda_{2}\right): u \in \Omega_{3}(a, b), \lambda_{1} \leq 0, \lambda_{2}=0\right\}, \\
& \Lambda_{4}(a, b)=\left\{\left(u, \lambda_{1}, \lambda_{2}\right): u \in \Omega_{4}(a, b), \lambda_{1} \leq 0, \lambda_{2} \leq 0\right\} .
\end{aligned}
$$

\subsection{A deformation lemma.}

Definition 2.1. Let $X$ be a Hilbert space, and $\eta: X \times \mathbb{R}^{+} \rightarrow X$ a continuous map. A subset $Y \subset X$ is positively invariant for $\eta$ if $\eta(Y, t) \subset Y \forall t \in \mathbb{R}^{+}$.

Theorem 2.1. Let $a, b \in \mathbb{R}, a<b$. Assume that

$$
\inf _{\Lambda(a, b)}\left\|\nabla J-\left(\lambda_{1} \nabla G_{1}-\lambda_{2} \nabla G_{2}\right)\right\|=\alpha>0
$$

where $\nabla G_{1}(u) \neq \mu \nabla G_{2}(u) \neq 0$ for all $\mu>0$, for every $u \in \Omega_{4}(a, b)$. Then, for all $a^{\prime}, b^{\prime} \in \mathbb{R}, a<a^{\prime}<b^{\prime}<b$ there exist a continuous map $\eta: \Omega(a, b) \times \mathbb{R}^{+} \rightarrow \Omega(a, b)$ and a constant $\rho>0$ such that:

(a) $\Omega\left(a^{\prime}, b^{\prime}\right) \cap\left\{G_{1} \leq 1\right\}$ is positively invariant under the action of $\eta$,

(b) $\Omega\left(a^{\prime}, b^{\prime}\right) \cap\left\{G_{2} \geq 1\right\}$ is positively invariant under the action of $\eta$,

(c) $\eta(u, t)=u \quad \forall t \in \mathbb{R}^{+}$if $u \in \partial \Omega(a, b)$,

(d) $J(\eta(u, \cdot))$ is decreasing $\forall u \in \Omega$, 
(e) $J(\eta(u, s))-J(\eta(u, t)) \geq \rho(t-s)$ whenever $\eta(u, r) \in \Omega\left(a^{\prime}, b^{\prime}\right) \cap\left\{G_{1} \leq\right.$ $1\} \cap\left\{G_{2} \leq 1\right\} \quad \forall r \in[s, t], \quad 0 \leq s<t$.

Proof. Step 1. Construction of a pseudogradient. Let $u \in \Omega(a, b)$ be fixed. From assumption (3) and elementary geometrical considerations, we obtain the existence of $w=w(u) \in H,\|w\|=\alpha$ such that

$$
\begin{aligned}
& \nabla J(u) w \geq \alpha^{2} / 2, \\
& \nabla G_{1}(u) w \geq 0 \quad\left(\neq 0 \text { if } G_{1}(u)>1 / 2\right), \\
& \nabla G_{2}(u) w \leq 0 \quad\left(\neq 0 \text { if } G_{2}(u)>1 / 2\right) .
\end{aligned}
$$

Now the proof is standard: by the regularity of $J, G_{1}$ and $G_{2}$, there exists $r=$ $r(u)>0$ such that the same properties (4) hold for all $v \in B_{r(u)}(u)$ with $v$ instead of $u$ and the same $w=w(u)$. Let us select a locally finite refinement $\mathcal{U}:=\left\{U_{i}\right\}_{i \in I}$ of $\left\{B_{r(u)}(u)\right\}_{u \in \Omega(a, b)}$ and a corresponding Lipschitz continuous partition of unity $\left\{p_{i}\right\}_{i \in I}$. Finally we define

$$
W(u)=\sum_{i \in I} p_{i}(u) w\left(u_{i}\right)
$$

It turns out that $W$ is a locally Lipschitz pseudogradient for $J$ and satisfies (4).

Step 2. Construction of a pseudogradient flow. Let

$$
\begin{aligned}
& B=\Omega\left(a^{\prime}, b^{\prime}\right) \cap\left\{G_{1} \leq 1\right\} \cap\left\{G_{2} \leq 1\right\}, \\
& A=\Omega(a, b) .
\end{aligned}
$$

Consider a Lipschitz continuous function $\psi: H \rightarrow[0,1]$ which is 1 on $B$ and vanishes on $A^{c}$. Take the pseudogradient vector field $W$ just built for $J$ and observe that $W$ is well defined over $A$. Now define the locally Lipschitz vector field $V: H \rightarrow H$ :

$$
V(u)=\left\{\begin{array}{cl}
-\psi(u) W(u) \cdot\|W(u)\|^{-1} & u \in \Omega(a, b) \backslash H_{0}, \\
0 & \text { otherwise, }
\end{array}\right.
$$

where $H_{0}=\left\{u \in \Omega(a, b): \nabla J(u)-\left(\lambda_{1} \nabla G_{1}(u)-\lambda_{2} \nabla G_{2}(u)\right)=0,\left(u, \lambda_{1}, \lambda_{2}\right) \in\right.$ $\Lambda(a, b)\}$. By the choice of $\psi, V$ is Lipschitz continuous throughout $H$. Moreover $\|V\| \leq 1$, and so it is uniformly bounded. Hence, for any $u \in H$, there exists a unique solution $\eta(u, \cdot)$, defined on $\mathbb{R}$, to the following Cauchy problem in $H$ :

$$
\left\{\begin{aligned}
\frac{d}{d t} \eta(u, t) & =V(\eta(u, t)), \\
\eta(u, 0) & =u .
\end{aligned}\right.
$$

It turns out that $\eta$ is continuous in $u$, differentiable in $t$ and (c) holds by definition of $\psi$. The invariance stated in (a), (b) follows by the properties (4) of the pseudogradient $W$. Properties (d), (e) are proved by computing

$$
\begin{aligned}
J(\eta(u, s))- & J(\eta(u, t)) \\
& =-\int_{t}^{s} \psi(\eta(u, r))\left\langle\nabla J(\eta(u, r)), W(\eta(u, r)) \cdot\|W(\eta(u, r))\|^{-1}\right\rangle d r .
\end{aligned}
$$

2.2. A variational principle. Let $\delta>0$ be fixed and consider the square $Q:=$ $[\delta, 1 / \delta]^{2 n}(n \in \mathbb{N})$; let $\gamma_{0}: Q \rightarrow H$ be a $C^{2}$ function such that

$-\gamma_{0}(Q) \subset\left\{G_{1} \leq 1 / 2\right\}$,

$-\gamma_{0}(\partial Q) \subset\{J<0\} \cup\left\{G_{2}>1\right\}$. 
Then let us consider the following minimax structure:

$$
\begin{aligned}
\Gamma & :=\left\{\gamma(\cdot)=\eta\left(t, \gamma_{0}(\cdot)\right): t \geq 0\right\}, \\
E & :=\left\{G_{1}<1\right\} \cap\left\{G_{2}<1\right\}, \\
T_{\gamma} & :=\left\{z=\left(z_{i}\right)_{i=1}^{2 n} \in Q: \gamma(z) \in \bar{E}\right\} .
\end{aligned}
$$

Assuming that $T_{\gamma} \neq \emptyset$ for all $\gamma \in \Gamma$, we define the following value:

$$
c:=\inf _{\gamma \in \Gamma} \max _{z \in T_{\gamma}} J(\gamma(z)) \in \mathbb{R} \cup\{-\infty\} .
$$

Moreover the following holds:

Theorem 2.2. If $T_{\gamma} \neq \emptyset$ for all $\gamma \in \Gamma$ and $c>0$, then there exists a sequence $\left\{u_{n}, \lambda_{1}^{n}, \lambda_{2}^{n}\right\} \in \Lambda(c+1, c-1)$ such that $u_{n} \in \bar{E}, J\left(u_{n}\right) \rightarrow c$ and $\| \nabla J\left(u_{n}\right)-$ $\left(\lambda_{1}^{n} \nabla G_{1}\left(u_{n}\right)-\lambda_{2}^{n} \nabla G_{2}\left(u_{n}\right)\right) \| \rightarrow 0$.

Proof. By contradiction, assume the existence of $0<\delta<c / 2$ such that

$$
\left(u, \lambda_{1}, \lambda_{2}\right) \in \Lambda(c-2 \delta, c+2 \delta) \Longrightarrow \| \nabla J(u)-\left(\lambda_{1} \nabla G_{1}(u)-\lambda_{2} \nabla G_{2}(u) \|>\delta .\right.
$$

Now set $a=c-2 \delta(>0), b=c+2 \delta, a^{\prime}=c-\delta, b^{\prime}=c+\delta$ and apply the deformation lemma; we have a flow $\eta$ and a positive $\rho$ (we can assume $\rho<\delta$ ) satisfying properties (a)-(e) therein. Let us now consider a suboptimal path $\gamma \in \Gamma$ such that

$$
\max _{T_{\gamma}} J \circ \gamma \leq c+\rho / 2
$$

Note that $\psi:=\eta(\gamma, 1)$ belongs to $\Gamma$ and $G_{1}(\psi(t))<1$ for all $t \in Q$ by property (a). Moreover, since $\{J<0\}$ and $\left\{G_{2}>1\right\}$ are invariant under the flow $\eta$ by (c), $\psi(\partial Q) \subset\{J<0\} \cup\left\{G_{2}>1\right\}$. Now, by the assumption $T_{\psi} \neq \emptyset$ and the definition of $c$, there exists $\xi \in T_{\psi}$ such that $J(\psi(\xi)) \geq c, G_{1}(\psi(\xi)) \leq 1, G_{2}(\psi(\xi)) \leq 1$. Set $u_{\gamma}:=\gamma(\xi)$ and note that the invariance properties in (a) and (b) imply $\eta\left(u_{\gamma}, t\right) \in E$ for all $t \in[0,1]$. Thus, since $J \circ \eta$ is decreasing in $t$ by $(\mathrm{d})$ :

$$
c+\delta>c+\rho / 2 \geq J\left(x_{\gamma}\right) \geq J\left(\eta\left(u_{\gamma}, t\right)\right) \geq J\left(\eta\left(u_{\gamma}, 1\right)\right)=J(\psi(\xi)) \geq c, \quad t \in[0,1] .
$$

This implies that $\eta\left(u_{\gamma}, t\right) \in \Omega\left(a^{\prime}, b^{\prime}\right) \cap\left\{G_{1} \leq 1\right\} \cap\left\{G_{2} \leq 1\right\}$ for all $t \in[0,1]$ and by property (e) we obtain

$$
J\left(\eta\left(u_{\gamma}, 1\right)\right)=J(\psi(\xi))<c-\rho / 2,
$$

in contradiction with the definition of $c$.

With the previous minimaxing procedure, we obtain a sequence of almost constrained critical points: to obtain the desired true critical point, a suitable compactness condition is needed as in the following:

Definition 2.2. Let $\left\{u_{n}, \lambda_{1}^{n}, \lambda_{2}^{n}\right\} \subset \Lambda(c-1, c+1)$ be any sequence with the following properties:

- $J\left(u_{n}\right) \rightarrow c$,

$-\nabla J\left(u_{n}\right)-\lambda_{1}^{n} \nabla G_{1}\left(u_{n}\right)+\lambda_{2}^{n} \nabla G_{2}\left(u_{n}\right) \rightarrow 0$.

We say that a functional $J \in C^{1}(H, \mathbb{R})$ satisfies the constrained Palais-Smale condition with respect to $G_{1}$ and $G_{2}$ at level $c \in \mathbb{R}$ (say $\operatorname{CPS}_{c}$ ) if $\left\{u_{n}\right\}$ is relatively compact in $H$.

Definition 2.3. We say that the constraint $\left\{G_{1} \leq 1\right\} \cap\left\{G_{2} \leq 1\right\}$ is admissible at level $c$ if there exist $a<c<b$ such that the existence of $\left(u, \lambda_{1}, \lambda_{2}\right) \in \Lambda(a, b)$ such that $\nabla J(u)-\lambda_{1} \nabla G_{1}(u)+\lambda_{2} \nabla G_{2}(u)=0$ implies $\lambda_{i}=0, i=1,2$. 
Thus we immediately obtain the following corollary:

Corollary 2.1. Under the assumptions of Theorem 2.2, if J satisfies $C P S_{c}$ at the minimax level $c$ and $\left\{G_{1} \leq 1\right\} \cap\left\{G_{2} \leq 1\right\}$ is an admissible constraint, then there exists $u \in\left\{G_{1}<1\right\} \cap\left\{G_{2}<1\right\}$ critical point for $J$ at level c.

Proof. Let $\left\{u_{n}, \lambda_{1}^{n}, \lambda_{2}^{n}\right\}$ be the sequence provided by Theorem [2.2. By assumption $\mathrm{CPS}_{c}$, such a sequence is relatively compact and thus (up to subsequences) it strongly converges to some limit $\left(u, \lambda_{1}, \lambda_{2}\right)$ such that $u \in\left\{G_{1} \leq 1\right\} \cap\left\{G_{2} \leq 1\right\}$ (by continuity of $G_{i}$ ), $\lambda_{i} \leq 0, i=1,2$, and (by continuity of $J$ and $\nabla J, \nabla G_{i}$ ) it turns out that $J(u)=c$ and $\nabla J(u)-\lambda_{1} \nabla G_{1}(u)+\lambda_{2} \nabla G_{2}(u)=0$. Since our constraint is admissible, according to the definition above, we know that $\lambda_{1}=\lambda_{2}=0$ and thus $\nabla J(u)=0$ : thus $u$ is a critical point for $J$.

\section{Applichtion to Fourth order problems}

Here and throughout the rest of this paper we shall always assume that $c \geq$ $-(\pi / T)^{2}$ and that $f$ fulfills assumptions $\left(f_{1}\right),\left(f_{2}\right),\left(f_{3}\right)$. Let $n>0$ be a fixed integer: we are going to show how the abstract results of Section 2 apply to prove the existence of a solution to problem (1) having exactly $2 n$ simple zeroes in $(0, T]$, as stated in Theorem 1.1.

We shall deal with variational solutions to (1), i.e. $u \in H:=H^{2}(0, T)$ with periodic boundary conditions that are critical points of the energy functional associated to (1):

$$
J(u):=\int_{0}^{T}\left(\frac{1}{2}\left|u^{\prime \prime}(t)\right|^{2}+\frac{c}{2}\left|u^{\prime}(t)\right|^{2}-F(t, u(t))\right) d t .
$$

It turns out that $J$ is well defined on $H$ and $J \in C^{1}(H, \mathbb{R})$. We notice that the condition $c \geq-(\pi / T)^{2}$ implies that the quadratic part of $J$ is nonnegative definite, even when $c<0$. Moreover it defines an equivalent norm over those functions in $H$ having at least one zero.

Notation. Throughout the paper we shall denote by $\|\cdot\|$ the semi norm $\|u\|=$ $\left(\int_{0}^{T}\left|u^{\prime \prime}(t)\right|^{2} d t\right)^{1 / 2}, u \in H$, and by $\|u\|_{p}$ the usual $L^{p}$ norm, $p \geq 1$. Let us recall that $H \hookrightarrow L^{p}$ for all $p \geq 1$ and $H \hookrightarrow C^{1}([0, T])$.

In all the computations $C>0$ will denote any positive constant we need not specify.

3.1. The constraints. In this section we interpret the nodal property that a function $u \in H$ has $2 n$ simple zeroes as a topological property, namely $u \in\left\{g_{1}<1, g_{2}<\right.$ $1\} \subset H$ for suitable functionals $g_{i}, i=1,2$. In our construction we introduce parameters $\varepsilon>0, \delta>0$ that will be fixed later on in the paper.

Let $u \in H$ and consider

$$
g_{1}(u)=\int_{0}^{T} h(u(t)) k\left(u^{\prime}(t)\right) d t
$$

where the auxiliary functions $h$ and $k$ are defined as follows: fixed $\delta>0, h: \mathbb{R} \rightarrow \mathbb{R}^{+}$ is a $C^{1}$ even function such that $h \equiv 1$ in $[-\delta / 2, \delta / 2], h \equiv 0$ if $|t| \geq \delta$ and $h$ is decreasing on $[\delta / 2, \delta]$. On the other side $k \in C^{2}(\mathbb{R} \backslash\{0\})$ is a nonnegative even function, $k \equiv 0$ if $|t| \geq \delta$ and $k^{\prime}(t) t<0$ if $0<|t|<\delta$. Moreover $k$ behaves as $k(t) \asymp t^{-2}$ in a neighborhood of the origin and is convex on the positive half-line (see Figure 1). 

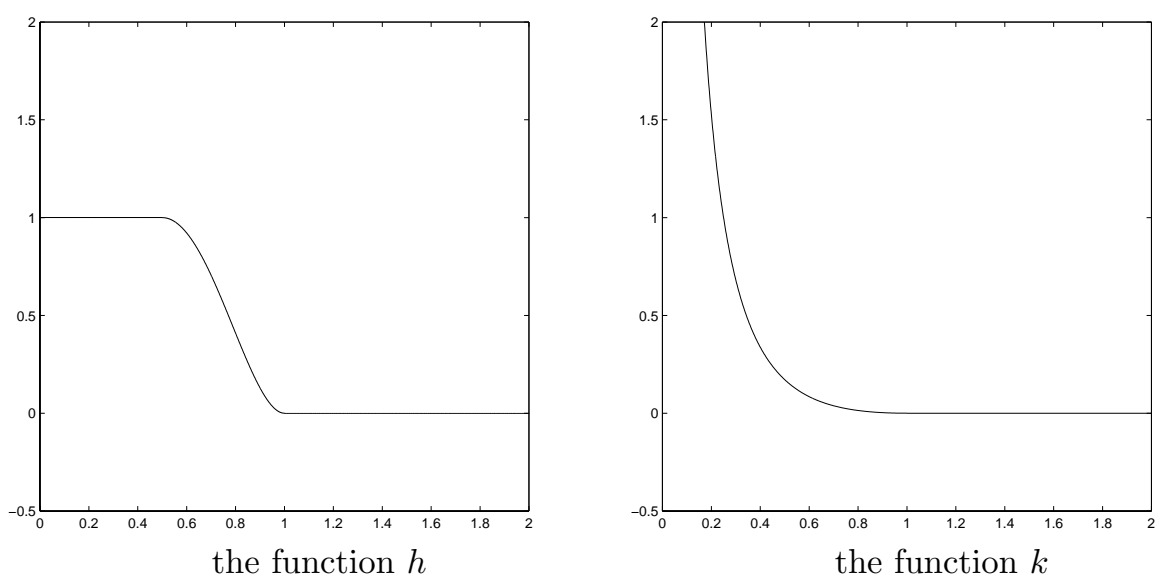

Figure 1. Possible graphs for $h$ and $k$, for $t>0$ and $\delta=1$.

As a first immediate consequence of such a behavior at the origin, $g_{1}$ satisfies the following property

Lemma 3.1. If $u \in H^{2}([0, T])$, then $g_{1}(u)<\infty$ implies

$$
\left|u^{\prime}(t)\right| \neq 0 \text { for all } t \text { such that }|u(t)|<\delta .
$$

Proof. Let $u \in H^{2}([0, T])$ with $g_{1}(u)<\infty$ and assume by contradiction the existence of an interval $[\alpha, \beta]$, where $\delta>\delta^{\prime} \geq u(t)>0, u^{\prime}(t)>0$ for all $t \in(\alpha, \beta]$ while $u^{\prime}(\alpha)=0$. Then, by construction, there exists $H>0$ (depending on $\delta^{\prime}$ ) such that $h(u(t)) \geq H$ for all $t \in[\alpha, \beta]$. Furthermore $k(s) \geq K\left(|s|^{-2}-1\right)$ for some positive $K$. Now, since $u \in H^{2} \hookrightarrow C^{1,1 / 2}$, there exists $C>0$ such that $u^{\prime}(t) \leq C(t-\alpha)^{1 / 2}$ and hence $k\left(u^{\prime}(t)\right) \geq C^{\prime}(t-\alpha)^{-1}-C^{\prime}$. Finally we obtain

$$
g_{1}(u) \geq H C^{\prime} \int_{\alpha}^{\beta} \frac{1}{t-\alpha} d t-T C^{\prime}=\infty .
$$

Hence, when $g(u)$ is finite, $u$ admits only nondegenerate zeroes and thus we can write

$$
\left\{g_{1}(u)<\infty\right\}=\bigcup_{n \in \mathbf{N}} \mathcal{A}_{n},
$$

where $\mathcal{A}_{n}$ is the set of $u \in H$ having $2 n$ simple zeroes in $(0, T]$.

Definition 3.1. Let $u \in \mathcal{A}_{n}$. We denote by $\left\{\tau_{i}\right\}_{i=1}^{2 n} \subset(0, T]$ the consecutive zeroes of $u$ in $(0, T]$ and we set $\tau_{i+2 k n}=\tau_{i}+k T$ for all $k \in \mathbb{Z}, i=1, \ldots, 2 n$. The $T$-periodic function $u_{i}(i=1, \ldots, 2 n)$ such that

$$
u_{i}(t)=\left\{\begin{array}{rc}
u(t), & t \in\left(\tau_{i-1}, \tau_{i}\right], \\
0, & t \in(0, T] \backslash\left(\tau_{i-1}, \tau_{i}\right],
\end{array}\right.
$$

is called the $i$-th bump of $u$. 
Let us now fix $\varepsilon>0$ and introduce the auxiliary functional $g_{2}: \mathcal{A}_{n} \rightarrow \mathbb{R}^{+}(n$ has been fixed above) as

$$
g_{2}(u)=\sum_{i=1}^{2 n} \varphi\left(\int_{0}^{T} \psi\left(u_{i}(t)\right) d t\right)
$$

where $\psi$ is a $C^{1}$ nonnegative function which is even and convex. Furthermore $\psi(v) \equiv 0$ if $0 \leq v<\varepsilon$, and there exist $C>0, q>1$ such that

$$
\psi(v) \geq\left|\frac{f(t, v)}{v}\right|^{q}-C \varepsilon .
$$

The second auxiliary function $\varphi: \mathbb{R}^{+} \rightarrow \mathbb{R}^{+}$is such that $\varphi(s)=0$ if $s \geq 10 \varepsilon$; moreover $\varphi \in C^{1}\left(\mathbb{R}^{+} \backslash\{0\}\right)$ is a decreasing function which is singular at the origin and satisfies

$$
\varphi(s) \approx-\varphi^{\prime}(s) s, \quad 0<s \leq 10 \varepsilon .
$$

As for $g_{1}$, a bound for $g_{2}$ implies some properties about the nodal structure of $u$ and, indirectly, of its derivatives. Indeed $g_{2}(u)<\infty$ implies that all the bumps have $L^{\infty}$ norm at least $\varepsilon$. On the other hand, when $g_{2}(u)$ is strictly positive, then there exists at least an integer $j$ such that $\int_{0}^{T} \psi\left(u_{j}\right) d t \leq 10 \varepsilon$.

We will need the auxiliary functionals $g_{1}$ and $g_{2}$ in the application of the abstract critical point results given in Section 2 To this aim a key point consists in proving the admissibility of the constraint $\left\{g_{1}(u)<1\right\} \cap\left\{g_{2}(u)<1\right\}$ (see Definition 2.3). As a matter of fact, this involves three steps: the first two for the study of the interaction between $\nabla J$ and $\nabla g_{i}$ separately on $g_{i}=1$, and the last involves the interaction at those points where both of the constraints activate.

Lemma 3.2. Let $u \in H$ such that $g_{1}(u) \in\left[\frac{1}{2}, 1\right]$ and $g_{2}(u) \leq 1$. Then

$$
\nabla J(u) \neq \lambda \nabla g_{1}(u), \quad \forall \lambda \leq 0,
$$

whenever $\delta>0$ is suitably smaller than $\varepsilon$.

Proof. Let $u \in H^{2}$ be a solution of (11). Then $u \in C^{4}(0, T)$ and it fulfills

$$
u^{i v}-c u^{\prime \prime}-f(t, u)=\lambda\left(-\frac{d}{d t}\left[h(u) k^{\prime}\left(u^{\prime}\right)\right]+h^{\prime}(u) k\left(u^{\prime}\right)\right) .
$$

Since $g_{2}(u) \leq 1$ we know that all the bumps are large $\left(\int_{0}^{T} \psi\left(u_{i}\right) d t>\varphi^{-1}(1)\right.$ and hence $\max \left|u_{i}\right|>c(\varepsilon)$ for all $\left.i\right)$; furthermore, since $g_{1}(u) \in\left[\frac{1}{2}, 1\right]$, we obtain the existence of $s_{0}$ such that $\left|u\left(s_{0}\right)\right|<\delta$ and $\left|u^{\prime}\left(s_{0}\right)\right|<\delta$ but $\left|u^{\prime}(t)\right| \neq 0$ for all $t$ such that $|u(t)|<\delta$. Let $s_{0} \in\left[\tau_{j-1}, \tau_{j}\right]$ and assume w.l.o.g. that $u_{j} \geq 0$ and $u^{\prime}\left(s_{0}\right)>0$.

If $\delta$ is very small compared to $\varepsilon$, then $s_{0}$ cannot be a point of absolute maximum for $u^{\prime}$ on $\left[s_{0}, t_{j}\right]$, otherwise $u_{j}$ could not reach its maximum, that is, larger than $\varepsilon$. Then there must exist a convex-concave inflection point of $u_{j}$ in $\left(s_{0}, t_{j}\right)$. The same kind of reasoning leads to the existence of a second convex-concave inflection point between $t_{j-1}$ (where $u\left(t_{j-1}\right)=\min u_{j-1}<-c$ ) and $s_{0}$. Hence we obtain the existence of a concave-convex inflection point between the previous two. We denote by $t_{j-1}<\alpha<\beta<t_{j}$ two consecutive inflection points with the property that $u \cdot u^{\prime \prime}>0 \in(\alpha, \beta)$. 
Now we multiply (12) by the test function $u^{\prime \prime}$ and then integrate between $\alpha$ and $\beta$ : the left-hand side becomes (after integration by parts, using $u^{\prime \prime}(\alpha)=u^{\prime \prime}(\beta)=0$ and the Poincaré inequality for $\left.u^{\prime \prime}\right)$

$$
-C \int_{\alpha}^{\beta}\left|u^{\prime \prime \prime}(t)\right|^{2} d t-\int_{\alpha}^{\beta} f(t, u(t)) u^{\prime \prime}(t) d t<0,
$$

where the inequality comes from the sign assumption $\left(f_{2}\right)$ and the fact that $u u^{\prime \prime}>0$ in $(\alpha, \beta)$. On the other hand, the right-hand side is $\lambda$ times

$$
\int_{\alpha}^{\beta}-h^{\prime}(u) k^{\prime}\left(u^{\prime}\right) u^{\prime} u^{\prime \prime}+\int_{\alpha}^{\beta}-h(u) k^{\prime \prime}\left(u^{\prime}\right)\left|u^{\prime}\right|^{2}+\int_{\alpha}^{\beta} h^{\prime}(u) k\left(u^{\prime}\right) u^{\prime \prime} .
$$

The second integral is obviously negative. Now recall how $h$ and $k$ are defined; in particular $h^{\prime}(v) v \leq 0$ and $k^{\prime}(v) v \leq 0$ for all $v$ and, since $u u^{\prime \prime}>0$, we realize that the first integral is negative. The same property of $h$ together with $k\left(u^{\prime}\right) \geq 0$ and again $u u^{\prime \prime}>0$ ensures that also the third integral is negative too and this, recalling that $\lambda \leq 0$, concludes the proof leading to a sign contradiction in identity (12).

Lemma 3.3. Let $u \in H$ such that $g_{2}(u) \in\left[\frac{1}{2}, 1\right]$ and $g_{1}(u) \leq 1$. Then

$$
\nabla J(u) \neq-\lambda \nabla g_{2}(u), \quad \forall \lambda \leq 0,
$$

whenever $\varepsilon>0$ is small enough.

Let $u \in H^{2}$ be a solution of (13). Then $u \in C^{4}(0, T)$ and it solves the equation

$$
u^{i v}-c u^{\prime \prime}-f(t, u)=-\lambda \sum_{i=1}^{2 n} \mu_{i} \psi^{\prime}\left(u_{i}\right),
$$

where $\mu_{i}=\varphi^{\prime}\left(\int_{0}^{T} \psi\left(u_{i}\right)\right)$ is negative when the bump $u_{i}$ is small (i.e. $\int_{0}^{T} \psi\left(u_{i}\right)<$ $10 \varepsilon)$ and $\mu_{i}=0$ otherwise. Observe that, since $g_{2}(u)$ is not 0 , there must exist an index $j$ such that $\mu_{j}<0$; we assume, to fix the ideas, that $u_{j} \geq 0$.

Now we define $\alpha$ according with the following rule: if $u^{\prime \prime}\left(\tau_{j-1}\right) \leq 0$, let $\alpha$ be the first inflection point to the left of $\tau_{j-1}$ (possibly, $\tau_{j-1}$ itself); if $u^{\prime \prime}\left(\tau_{j-1}\right)>0$, let $\alpha$ be the first inflection point to the right of $\tau_{j-1}$. Therefore, by construction, $\alpha$ is an increasing convex-concave inflection point, and in particular $u^{\prime}(\alpha)>0, u^{\prime \prime}(t)<0$ on a right neighborhood of $\alpha$. Now three cases may occur:

Case 1. $\mu_{j-1}=\mu_{j+1}=0$.

In this situation we choose a second inflection point near $\tau_{j}$ in an analogous way: if $u^{\prime \prime}\left(\tau_{j}\right) \leq 0$ let $\beta$ be the first inflection point on the right of $\tau_{j}$ (possibly, $\tau_{j}$ itself). If not, let $\beta$ be the first inflection point on the left of $\tau_{j}$. We obtain that $u^{\prime}(\beta)<0, u^{\prime \prime}(t)<0$ on a left neighborhood of $\beta$ (and it is an inflection point of concave-convex type). Let us multiply (14) by $u^{\prime \prime}$ and integrate in $[\alpha, \beta]$. We wish to show that the left-hand side is negative while the right-hand side is positive, leading to a contradiction. Let us first study the left-hand side

$$
-\int_{\alpha}^{\beta}\left|u^{\prime \prime \prime}\right|^{2} d t-c \int_{\alpha}^{\beta}\left|u^{\prime \prime}(t)\right|^{2} d t-\int_{\alpha}^{\beta} f(t, u) u^{\prime \prime} d t .
$$

Notice that, by $\left(f_{2}\right)$ and Poincaré inequality applied to $u^{\prime \prime}$, this is certainly negative on the intervals where $u u^{\prime \prime} \geq 0$. By construction, if $\alpha<\tau_{j-1}$ (resp. $\beta>\tau_{j}$ ) we have 
that $u u^{\prime \prime} \geq 0$ on $\left[\alpha, \tau_{j-1}\right]$ (resp. on $\left.\left[\tau_{j}, \beta\right]\right)$. Hence we can restrict our attention to the interval $I:=\left[\max \left\{\alpha, \tau_{j-1}\right\}, \min \left\{\beta, \tau_{j}\right\}\right]$. We estimate as follows:

$$
\int_{I}\left|\frac{f(t, u)}{u} u u^{\prime \prime}\right| d t \leq\left(\int_{I}\left|u^{\prime \prime}\right|^{p}\right)^{\frac{1}{p}}\left(\int_{I}|u|^{p}\right)^{\frac{1}{p}}\left(\int_{I}\left|\frac{f(t, u)}{u}\right|^{q}\right)^{\frac{1}{q}},
$$

where $\frac{2}{p}+\frac{1}{q}=1$. The last integral is small with $\varepsilon$, since by construction it holds

$$
\int_{I}\left|\frac{f(t, u)}{u}\right|^{q} \leq \int_{\tau_{j-1}}^{\tau_{j}}\left(\psi\left(u_{j}\right)+C \varepsilon\right) \leq(10+T C) \varepsilon .
$$

We assert that there exists $C>0$, not depending on $\varepsilon$, such that

$$
\int_{I}|u|^{p} \leq C \int_{I}\left|u^{\prime \prime}\right|^{p}
$$

This inequality is completely standard if both $u$ and $u^{\prime}$ annihilate somewhere in $I$, that is, if either $\alpha$ or $\beta$ belong to $I$. The only missing case is when $u^{\prime \prime}\left(\tau_{j-1}\right)>0$, $u^{\prime \prime}\left(\tau_{j}\right)>0$ and thus $I=[\alpha, \beta] \subset\left[\tau_{j-1}, \tau_{j}\right]$. In such a situation, we consider a $C^{1}$ auxiliary function $\tilde{u}$ that is the smallest concave regular function greater than $u$ on $[\alpha, \beta]$, and we define $\tilde{u}$ to be linear in $\left[\alpha^{\prime}, \alpha\right]$ and in $\left[\beta, \beta^{\prime}\right]$ for some $\alpha^{\prime}<\alpha$ and $\beta^{\prime}>\beta$ where $\tilde{u}\left(\alpha^{\prime}\right)=\tilde{u}\left(\beta^{\prime}\right)=0$. Note that by construction $\left[\alpha^{\prime}, \beta^{\prime}\right] \subset\left[\tau_{j-1}, \tau_{j}\right]$, since $u$ is convex on $\left[\tau_{j-1}, \alpha\right] \cup\left[\beta, \tau_{j}\right]$. Now the Poincaré and Sobolev inequalities for $\tilde{u}$ give

$$
\int_{[\alpha, \beta]}|u|^{p} \leq \int_{\left[\alpha^{\prime}, \beta^{\prime}\right]}|\tilde{u}|^{p} \leq C\left(\alpha^{\prime}, \beta^{\prime}\right) \int_{\left[\alpha^{\prime}, \beta^{\prime}\right]}\left|\tilde{u}^{\prime \prime}\right|^{p}=C\left(\alpha^{\prime}, \beta^{\prime}\right) \int_{[\alpha, \beta]}\left|u^{\prime \prime}\right|^{p}
$$

where in fact $C\left(\alpha^{\prime}, \beta^{\prime}\right) \leq C(0, T)$ is bounded.

Inequality (17) and the Poincaré inequality applied to $u^{\prime \prime}$ allows us to estimate from above the left-hand side with

$$
-C \int_{\alpha}^{\beta}\left|u^{\prime \prime \prime}\right|^{2} d t+C \varepsilon\left(\int_{\alpha}^{\beta}\left|u^{\prime \prime \prime}\right|^{2}\right)
$$

and this is finally negative if $\varepsilon$ is small enough.

Coming to the right-hand side, we wish to show that

$$
\sum_{i=1}^{2 n} \mu_{i} \int_{\alpha}^{\beta} \psi^{\prime}\left(u_{i}\right) u^{\prime \prime} \geq 0
$$

where the $\mu_{j}$ 's are nonpositive. Since $\mu_{j-1}=\mu_{j+1}=0$, the sum actually consists of one single term, namely the one with $i=j$. Integrating by parts we find that

$$
\int_{\max \left\{\alpha, \tau_{j-1}\right\}}^{\min \left\{\beta, \tau_{j}\right\}} \psi^{\prime}\left(u_{j}\right) u^{\prime \prime}=\left[\psi^{\prime}\left(u_{j}\right) u^{\prime}\right]_{\max \left\{\alpha, \tau_{j-1}\right\}}^{\min \left\{\beta, \tau_{j}\right\}}-\int_{\max \left\{\alpha, \tau_{j-1}\right\}}^{\min \left\{\beta, \tau_{j}\right\}} \psi^{\prime \prime}\left(u_{j}\right)\left|u^{\prime}\right|^{2},
$$

and this is certainly negative since by construction $\psi^{\prime}\left(u_{j}(t)\right) \geq 0$ while $u^{\prime}(\beta), u^{\prime}\left(\tau_{j}\right)$ are negative and $u^{\prime}(\alpha), u^{\prime}\left(\tau_{j-1}\right)$ are positive.

Therefore Case 1 is worked out. The other two cases will be proven with suitable minor changes.

Case $2: \mu_{j-1} \neq 0$ or $\mu_{j+1} \neq 0$, but there exists at least one $l$ such that $\mu_{l}=0$.

Since not every $\mu_{i}$ is different from 0 , we can assume without loss of generality that $\mu_{j-1}=0, \mu_{j}, \mu_{j+1}, \ldots, \mu_{j+h}<0, \mu_{j+h+1}=0$ with $h \geq 1$. As before, we 
choose a second inflection point in the following way: if $u^{\prime}\left(\tau_{j+h}\right) u^{\prime \prime}\left(\tau_{j+h}\right) \geq 0$ let $\beta$ be the first inflection on the right of $\tau_{j+h}$; if $u^{\prime}\left(\tau_{j+h}\right) u^{\prime \prime}\left(\tau_{j+h}\right)<0$ let $\beta$ be the first inflection on the left of $\tau_{j+h}$. We multiply (14) by $u^{\prime \prime}$ and integrate on $[\alpha, \beta]$. Then the arguments already used in Case 1 apply to the following remarks: an inequality like (16) holds for every bump such that $\mu_{i} \neq 0$; an inequality like (17) holds since both $u$ and $u^{\prime}$ annihilate somewhere on $[\alpha, \beta]$; finally, the right-hand side writes $-\lambda$ (that is, nonnegative) times

$$
\begin{aligned}
\mu_{j} \int_{\max \left\{\alpha, \tau_{j-1}\right\}}^{\tau_{j}} \psi^{\prime}\left(u_{j}\right) u^{\prime \prime}+\sum_{i=j+1}^{j+h-1} \mu_{i} \int_{\tau_{i-1}}^{\tau_{i}} \psi^{\prime}\left(u_{i}\right) u^{\prime \prime} & \\
& +\mu_{j+h+1} \int_{\tau_{j+h-1}}^{\min \left\{\beta, \tau_{j+h}\right\}} \psi^{\prime}\left(u_{j+h+1}\right) u^{\prime \prime}
\end{aligned}
$$

and again, integrating by parts and recalling the signs of $\psi^{\prime}\left(u_{i}(t)\right)$ and $u^{\prime}(t)$ in the extremal points, we can conclude

Case 3: $\mu_{i} \neq 0$ for every $i$.

In this situation we choose $\beta:=\alpha+T$. Repeating again the arguments of Cases 1 and 2 one can easily conclude.

Lemma 3.4. Let $u \in H$ such that $g_{1}(u) \in\left[\frac{1}{2}, 1\right]$ and $g_{2}(u) \in\left[\frac{1}{2}, 1\right]$. Then

$$
\nabla J(u) \neq \lambda_{1} \nabla g_{1}(u)-\lambda_{2} \nabla g_{2}(u) \quad \forall \lambda_{1} \leq 0, \lambda_{2} \leq 0
$$

if $\varepsilon>0$ is small enough and $\delta$ is suitably smaller than $\varepsilon$.

Proof. As a preliminary remark, we observe that the arguments in the proofs of Lemmas 3.2 and 3.3 apply also to the present situation on suitable intervals where either $\nabla g_{1}(u)$ or $\nabla g_{2}(u)$ vanish. More precisely, we recall that if an interval $[a, b]$ is such that either $|u(t)| \leq \varepsilon$ on that interval, or $[a, b] \subset \operatorname{supp}\left(u_{i}\right)$ with $\mu_{i}:=$ $\varphi^{\prime}\left(\int_{0}^{T} \psi\left(u_{i}\right)\right)=0$, the sole constraint related to $g_{1}$ activates. On the other hand, on intervals $[a, b]$ such that $\max \left\{|u(t)|,\left|u^{\prime}(t)\right|\right\} \geq \delta$, only $g_{2}(u)$ is nonzero. To prove the lemma, we assume by contradiction that the equation corresponding to (18) has a solution $u \in C^{4}$. We will show that it is always possible to find an interval on which the arguments of one of the previous lemmas apply straightforward.

Since $g_{1}(u) \in[1 / 2,1]$ we have that $\left|u^{\prime}(t)\right| \neq 0$ for all $t$ such that $|u(t)|<\delta$, and there exists $s_{0}$ such that $\left|u\left(s_{0}\right)\right|<\delta$ and $\left|u^{\prime}\left(s_{0}\right)\right|<\delta$. Moreover, since $g_{2}(u) \in$ $[1 / 2,1]$, then for every $i$ we have $\max \left|u_{i}\right|>\varepsilon$, and there exists at least a bump, say $u_{j}$, such that $\int_{0}^{T} \psi\left(u_{j}\right)<10 \varepsilon$.

(Construction of $\alpha$ ) Denote by $I$ the interval such that $s_{0} \in I$ and $|u(t)|<\delta$; since $u^{\prime} \neq 0$ therein, we can assume that $u^{\prime}(t)>0$ for all $t \in I$. By elementary considerations, taking $\delta$ suitably smaller than $\varepsilon$, it follows that there exists a concave-convex inflection point $\alpha$ such that $|u(\alpha)|<(1+\delta) T$ and $\left|u^{\prime}(\alpha)\right|<\delta$ (otherwise $|u|$ could not reach its maximum at a level larger than $\varepsilon$ ). Let us assume that $u(\alpha)>0$. Observe that, if $\alpha \in \operatorname{supp}\left(u_{h}\right)$ and $\mu_{h}=0$, then, arguing as in Lemma 3.2, we can choose a second inflection point $\beta>\alpha$ with the property that $u \cdot u^{\prime \prime}>0$ on $(\alpha, \beta)$ and testing with $u^{\prime \prime}$ on $[\alpha, \beta]$ we obtain a contradiction. Hence we can assume $\alpha \in\left[\tau_{j-1}, \tau_{j}\right]$, where $j$ is as above, and, to fix the ideas, $u_{j}(t) \leq C \delta$ on $\left[\tau_{j-1}, \alpha\right]$.

(Construction of $\beta$ ) Let $t_{j} \in\left[\tau_{j-1}, \tau_{j}\right]$ such that $u\left(t_{j}\right)=\max \left(u_{j}(t)\right.$ ) (if the maximum is not uniquely achieved, choose the point nearest to $\tau_{j-1}$ ). Clearly 
$t_{j}>\alpha, u_{j}\left(t_{j}\right)>\varepsilon$ and $u_{j}^{\prime}\left(t_{j}\right)=0$. Now let $\rho$ be the unique point in $\left[\alpha, t_{j}\right]$ such that $u_{j}(\rho)=\delta$ when such a point exists (i.e., if $u(\alpha)<\delta$ ), otherwise let $\rho=\alpha$. Consider the problem $\max \left\{u_{j}^{\prime}(t): t \in\left[\rho, t_{j}\right]\right\}$. There are two possibilities: if this maximum is achieved in $\rho$, then $u^{\prime \prime}(\rho) \leq 0$, and we define $\beta$ as the first inflection point to the left of $\rho$ (possibly $\rho$ itself); if the maximum is achieved in an internal inflection point, let $\beta$ be such a point. In any case $\beta>\alpha, u^{\prime}(\beta)>\varepsilon / T, u^{\prime \prime}(\beta)=0, \beta$ is an increasing convex-concave inflection point and, as the reader can easily check, $\nabla g_{1}(u)$ is identically zero on $\left[\beta, t_{j}\right]$. We claim that

$$
\left|\frac{u(\beta)}{u^{\prime}(\beta)}\right| \leq 2 T \text {. }
$$

Indeed, by the Mean Value Theorem, $\max _{\left[\rho, t_{j}\right]} u^{\prime}(t)>\left(u\left(t_{j}\right)-u(\rho)\right) /\left(t_{j}-\rho\right)$, and $t_{j}-\rho<T, u(\beta)<u\left(t_{j}\right), u(\rho)=\delta$.

(Construction of $\gamma$ ) Now we are going to find a third inflection point $\gamma>\beta$, such that $\nabla g_{1}(u)$ is identically zero on $[\beta, \gamma]$, and such that testing (18) with $u^{\prime \prime}$ on $[\beta, \gamma]$ we will obtain rightly the same contradiction as in the proof of Lemma 3.3. Roughly speaking, to find $\gamma$, we increase $t$ on the right of $t_{j}$ until $g_{2}$ turns off or $g_{1}$ turns on. Again we have two possibilities. If there exists $h \geq 1$ such that $\mu_{j+h}:=\varphi^{\prime}\left(\int_{0}^{T} \psi\left(u_{j+h}\right)\right)=0, \mu_{j+i} \neq 0$ for $i<h$ and $\min \left\{|u(t)|,\left|u^{\prime}(t)\right|\right\} \geq \delta$ for $t \in\left[\beta, t_{j+i}\right]$ (where $t_{j+i}$ is the first maximum of $u_{j+i}$ ), then we can argue exactly as in Lemma 3.3, choosing $\gamma$ according with the sign of $u^{\prime}\left(\tau_{j+i-1}\right) u^{\prime \prime}\left(\tau_{j+i-1}\right)$, and obtaining a contradiction (the only minor difference is that, to obtain the Poincaré type inequality (17) as in Case 1 there, we convexify only on the right of $\gamma$ and not on the left of $\beta$ ). If, on the other hand, there exists $s_{1}$ such that $s_{1}>t_{j}$, $\max \left\{\left|u\left(s_{1}\right)\right|,\left|u^{\prime}\left(s_{1}\right)\right|\right\} \leq \delta, \min \left\{|u(t)|,\left|u^{\prime}(t)\right|\right\}>\delta$ on $\left[\beta, s_{1}\right)$, and $s_{1} \in\left[\tau_{j+h-1}, \tau_{j+h}\right]$ with $\mu_{j+i} \neq 0$ for $i \leq h$, then we can argue as above for the construction of $\alpha$ and $\beta$ and define $\gamma$ as that inflection point on the left of $s_{1}$ with $\nabla g_{1}(u)$ identically zero on $[\beta, \gamma]$ and $|u(\gamma)| /\left|u^{\prime}(\gamma)\right| \leq 2 T$. One can test the equation with $u^{\prime \prime}$ on $[\beta, \gamma]$ as in Lemma 3.3 , and repeat the same estimates. Again, the only difference consists in obtaining the Poincaré type inequality. As a consequence, the lemma will be proved if we can show that the following inequality (see (17)) holds: there exists $C>0$, not depending on $\varepsilon$ and $\delta$, such that

$$
\int_{[\beta, \gamma]}|u|^{p} \leq C \int_{[\beta, \gamma]}\left|u^{\prime \prime}\right|^{p} .
$$

To prove it we proceed as in Lemma 3.3 we consider a $C^{1}$ auxiliary function $\tilde{u}$ that is the smallest concave regular function greater than $u$ on $[\beta, \gamma]$, and that is linear in $\left[\beta^{\prime}, \beta\right]$ and in $\left[\gamma, \gamma^{\prime}\right]$ for some $\beta^{\prime}<\beta$ and $\gamma^{\prime}>\gamma$, where $\tilde{u}\left(\beta^{\prime}\right)=\tilde{u}\left(\gamma^{\prime}\right)=0$. Since the Poincaré inequality holds for $\tilde{u}$, we obtain

$$
\int_{[\beta, \gamma]}|u|^{p} \leq \int_{\left[\beta^{\prime}, \gamma^{\prime}\right]}|\tilde{u}|^{p} \leq C\left(\beta^{\prime}, \gamma^{\prime}\right) \int_{\left[\beta^{\prime}, \gamma^{\prime}\right]}\left|\tilde{u}^{\prime \prime}\right|^{p}=C\left(\beta^{\prime}, \gamma^{\prime}\right) \int_{[\beta, \gamma]}\left|u^{\prime \prime}\right|^{p},
$$

where the constant $C\left(\beta^{\prime}, \gamma^{\prime}\right)$ is bounded if and only if $\beta-\beta^{\prime}=u(\beta) / u^{\prime}(\beta)$ and $\gamma^{\prime}-\gamma=|u(\gamma)| /\left|u^{\prime}(\gamma)\right|$ are bounded. But this is true by (19) and by the respective inequality in $\gamma$. Thus inequality (20) holds and the proof of the lemma can be completed. 
From now on we assume that $\varepsilon$ and $\delta$ are chosen in such a way that all the previous lemmas hold.

Remark 3.1. As a consequence of the lemmas above we derive that the constraint $\left\{g_{1}(u) \leq 1, g_{2}(u) \leq 1\right\}$ is admissible according to Definition 2.3 and the assumptions of the deformation lemma in Section 2 are satisfied by $G_{1}:=g_{1}, G_{2}=: g_{2}$ and $J$. Moreover, by Lemma 3.1 if $u \in\left\{g_{1}(u) \leq 1, g_{2}(u) \leq 1\right\}$, then $u$ has exactly $2 n$ simple zeroes.

3.2. The minimax structure. In this section we show that the variational principle introduced in Section 2.2 is applicable in our framework. To this aim, let $Q:=[\delta, 1 / \delta]^{2 n}(n \in \mathbb{N})$ : we are going to define a $C^{2}$ function, named $\gamma_{0}: Q \rightarrow \mathcal{A}_{n}$, such that

(a) $\gamma_{0}(Q) \subset\left\{g_{1} \leq 1 / 2\right\}$,

(b) $\gamma_{0}(\partial Q) \subset\{J<0\} \cup\left\{g_{2}>3 / 2\right\}$.

Let us first divide $(0, T)$ in $2 n$ subintervals $I_{j}=\left[x_{j}, x_{j+1}\right]$ of equal length and each $I_{j}$ again in three smaller subintervals of length $T /(6 n)$ by letting $x_{j}<y_{1, j}<y_{2, j}<$ $x_{j+1}$.

Now let $z=\left(z_{1}, \ldots, z_{2 n}\right) \in Q$ be given and define $\gamma_{0}$ as follows:

$$
\gamma_{0}(z)(t)=\left\{\begin{array}{lc}
(-1)^{j-1} z_{j}, & t \in\left\{y_{1, j}, y_{2, j}\right\}, \\
P_{j}(t), & t \in\left(y_{1, j}, y_{2, j}\right), \\
\text { piecewise linear, } & \text { otherwise, }
\end{array}\right.
$$

where $P_{j}$ is a second degree polynomial function such that $\left\|P_{j}\right\|_{C^{1}\left(\left[y_{1, j}, y_{2, j}\right]\right)} \leq$ $10(1+T)^{-1}|z|, j=1, \ldots, 2 n$, and the resulting function $\gamma_{0}$ is of class $C^{1}$.

Then by construction $\gamma_{0}$ has the following properties:

- if $\exists i$ such that $z_{i}=\delta$, then the $i$-th bump $\gamma_{0_{i}}(z)$ is small since $\max \left|\gamma_{0_{i}}\right|<$ $2 \delta<\varepsilon$ and thus $g_{2}\left(\gamma_{0}(z)\right)=\infty$.

- if $\exists i$ such that $z_{i}=1 / \delta$ and $\delta$ is small enough, then $J\left(\gamma_{0}(z)\right)<0$. In fact $\left\|\gamma_{0}\right\|>\left\|\gamma_{0 i}\right\| \asymp z_{i} \rightarrow \infty$ if $\delta \rightarrow 0$; since by the superlinearity condition $\left(f_{1}\right)$ it turns out that $J(u) \rightarrow-\infty$ if $\|u\| \rightarrow \infty$ on finite-dimensional spaces, then the desired inequality is proved.

Summarizing, we have

Lemma 3.5. If $\delta$ is small enough, then

(a) $u \in \gamma_{0}(\partial Q) \Rightarrow u \in\{J(u)<0\} \cup\left\{g_{2}(u)>3 / 2\right\}$;

(b) $u \in \gamma_{0}(Q) \Rightarrow g_{1}(u)<1$.

Now, according to Section 2 we consider the deformation $\eta$ provided by the deformation lemma with the choice for the constrain functionals $G_{1}=g_{1}$ and $G_{2}=g_{2}$. Then we define a minimax structure as in Section 2.2.

$$
\begin{aligned}
\Gamma & :=\left\{\gamma(\cdot)=\eta\left(\gamma_{0}(\cdot), t\right): t \geq 0\right\}, \\
E & :=\left\{G_{1}<1, G_{2}<1\right\}, \\
T_{\gamma} & :=\{z \in Q: \gamma(z) \in \bar{E}\}, \\
c & :=\inf _{\gamma \in \Gamma \in T_{\gamma}} J(\gamma(z)) .
\end{aligned}
$$

We claim that:

Lemma 3.6. $T_{\gamma} \neq \emptyset$ for all $\gamma \in \Gamma$ and $c>0$. 
First we need an auxiliary result.

Lemma 3.7. Let $\alpha>0$. Then

$$
\inf \left\{J(u): u \in \mathcal{A}_{n}, \int_{0}^{T} \psi\left(u_{i}(t)\right) d t=\alpha, \quad i=1, \ldots, 2 n\right\}>0
$$

if $\alpha$ and $\varepsilon / \alpha$ are small enough.

Proof. Let $u \in H$ such that $\int_{0}^{T} \psi\left(u_{i}(t)\right) d t=\alpha$ for all $i$ : by the properties of $\psi$ as in (91), we deduce that

$$
\int_{0}^{T}\left|\frac{f\left(u_{i}\right)}{u_{i}}\right|^{q} \leq \alpha+C \varepsilon, \quad i=1, \ldots, 2 n .
$$

By $\left(f_{1}\right)$ we can estimate $J$ as follows:

$$
J(u) \geq \frac{1}{2} \int_{0}^{T}\left(\left|u^{\prime \prime}\right|^{2}+c\left|u^{\prime}\right|^{2}-2 F(u)\right) \geq \frac{1}{2} \int_{0}^{T}\left(\left|u^{\prime \prime}\right|^{2}-\frac{2}{2+\gamma} f(u) u\right) .
$$

Now by the Holder inequality with $q$ and $p=q^{\prime}$

$$
\int_{0}^{T} f(u) u \leq\left(\int_{0}^{T}\left|\frac{f(u)}{u}\right|^{q}\right)^{1 / q}\left(\int_{0}^{T} u^{2 p}\right)^{1 / p}
$$

and thus, since $\|u\|=\left(\int_{0}^{T}\left|u^{\prime \prime}\right|^{2}\right)^{1 / 2}$ is an equivalent norm in $\mathcal{A}_{n}$,

$$
J(u) \geq C\|u\|^{2}-\frac{1}{2+\gamma}(\alpha+C \varepsilon)^{1 / q}\|u\|_{2 p}^{2} \geq\|u\|^{2}\left(C-\frac{S}{2+\gamma}(\alpha+C \varepsilon)^{1 / q}\right),
$$

where $S$ is the Sobolev constant giving the embedding $H \subset L^{2 p}$. Now the proof is done with $\varepsilon$ and $\alpha$ small enough to give positive sign to the right-hand side.

Proof of Lemma 3.6. Let $\gamma \in \Gamma$ be fixed, i.e. $\gamma=\eta\left(s, \gamma_{0}\right)$ for some fixed $s>$ 0 . By following the evolution under $\eta$ of the simple zeroes of $\gamma_{0}$ we may write $\gamma=\sum_{i=1}^{2 n} \sigma_{i}\left(\eta\left(s, \gamma_{0}\right)\right)_{i}$ in a continuous way (with respect to $s$ ). Therefore this decomposition induces a continuous map:

$$
\begin{aligned}
\mathcal{H}_{\gamma}: \quad[\delta, 1 / \delta]^{2 n} & \rightarrow \mathbb{R}^{2 n}, \\
z=\left(z_{i}\right)_{i=1}^{2 n} & \mapsto\left(\int_{0}^{T} \psi\left(\left(\eta\left(\gamma_{0}(z)(t), s\right)\right)_{i}\right) d t\right)_{i=1}^{2 n} .
\end{aligned}
$$

Now let $z \in \partial Q$ (i.e. there exists $i$ such that $z_{i} \in\{\delta, 1 / \delta\}$ ); by construction it holds:

- if $z_{i}=\delta$, then the $i$-th component of $\mathcal{H}_{\gamma}$ is zero (since by the invariance of $\left\{g_{2}>1\right\}$ it holds $\eta(s, \gamma(z))=\gamma(z)$ for all $\left.s>0\right)$;

- if $z_{i}=1 / \delta$, then the $i$-th component of $\mathcal{H}_{\gamma}$ is very large as soon as $\delta$ is small enough (here we are using the invariance of $\{J<0\}$ ).

Since this holds for all $i=1, \ldots, 2 n$, we are in a position to apply Miranda's Theorem [12]. As a consequence, if we fix $\alpha \in(0,1)$ we find $\bar{z}=\left(\bar{z}_{i}\right)_{i=1}^{2 n} \in Q$ such that $\mathcal{H}_{\gamma}(\bar{z})=(\alpha)_{i=1}^{2 n}$. This means

$$
\int_{0}^{T} \psi\left(\gamma_{i}(\bar{z})(t)\right) d t=\alpha, \quad \forall i=1, \ldots, 2 n,
$$


and thus, having care to choose $\varepsilon$ from the beginning small enough that $\varphi(\alpha)<$ $1 / 2 n$, we find

$$
g_{2}\left(\gamma_{i}(\bar{z})\right)=\sum_{i=1}^{2 n} \varphi(\alpha)<1 .
$$

If, moreover, $\alpha$ is chosen in such a way to satisfy the requirements of Lemma 3.7 we have

$$
J(\gamma(\bar{z}))>0
$$

To conclude the proof of the lemma it suffices to note that, by the invariance of $\left\{g_{1}<1\right\}$ under the action of $\eta$, it holds that $g_{1}(\gamma(\bar{z})) \leq 1$ and finally $\bar{z} \in T_{\gamma}$. The assertion $c>0$ now follows by (22).

Now we are in a position to apply Theorem 2.2. in order to find a critical point, however, we still have to check the constraint Palais-Smale condition $\mathrm{CPS}_{c}$ as stated in Corollary 2.1 for, as we already noticed in Remark 3.1 the used constraint is admissible (in the sense of Definition 2.3). Unfortunately, at this stage, the CPS condition does not hold, for we are not able to prove the boundedness of the $\mathrm{CPS}_{c}$ sequences. The aim of the following section is to prove some weaker type of compactness that will allow us to conclude the proof anyway.

3.3. Compactness properties. Let us fix $c \in \mathbb{R}$ and $a>0$. Let us define

$$
G_{1}(u)=g_{1}(u), \quad G_{2}(u)=g_{2}(u)-a[J(u)-c]
$$

and consider a $\mathrm{CPS}_{c}$ sequence (constrained with respect to $G_{1}$ and $G_{2}$ ), that is, $\left\{u_{n}, \lambda_{1}^{n}, \lambda_{2}^{n}\right\} \in \Lambda(c-1, c+1)$ such that $\left\{J\left(u_{n}\right)\right\} \rightarrow c$ and

$$
\nabla J\left(u_{n}\right)-\lambda_{1}^{n} \nabla G_{1}\left(u_{n}\right)+\lambda_{2}^{n} \nabla G_{2}\left(u_{n}\right) \rightarrow 0 .
$$

Lemma 3.8. For all $a>0$, the functional $J$ satisfies the constrained Palais-Smale conditions with respect to $G_{1}$ and $G_{2}$ at level $c$ in $\mathcal{A}_{n}$ if $n \geq 1$.

Proof. Let us first prove that $\left\{u_{n}\right\} \subset \mathcal{A}_{n}$ is bounded in $H$. Notice that in this subset of $H\left\|u^{\prime \prime}\right\|_{2}$ gives an equivalent norm. Let us multiply (23) at fixed $k$ by $u_{k}$ :

$$
\left(1-a \lambda_{2}^{k}\right) \nabla J\left(u_{k}\right) u_{k}-\lambda_{1}^{k} \nabla g_{1}\left(u_{k}\right) u_{k}+\lambda_{2}^{k} \nabla g_{2}\left(u_{k}\right) u_{k}=o(1)\left\|u_{k}\right\| .
$$

Note that the coefficient of $\nabla J$ is greater than 1 , so we divide by $1-a \lambda_{2}^{k}$ and we obtain

$$
\nabla J\left(u_{k}\right) u_{k}-\mu_{1}^{k} \nabla g_{1}\left(u_{k}\right) u_{k}+\mu_{2}^{k} \nabla g_{2}\left(u_{k}\right) u_{k}=o(1)\left\|u_{k}\right\|,
$$

where the sequence $\mu_{2}^{k}$ is bounded.

By assumption $\left(f_{1}\right)$ and the definition of $J$, one obtains

$$
\nabla J\left(u_{k}\right) u_{k} \leq \frac{\gamma}{2}\left\|u_{k}\right\|^{2}+C_{k}
$$

where $C_{k}$ is a bounded sequence; moreover $\nabla g_{1}\left(u_{k}\right) u_{k}<0$ for all $n$ by the sign properties of $h, k$ and their derivatives. Finally $\nabla g_{2}\left(u_{k}\right) u_{k}$ is bounded since by property (10) it holds that $\nabla g_{2}\left(u_{k}\right) u_{k} \approx g_{2}\left(u_{k}\right)$ and $g_{2}\left(u_{k}\right) \leq 3 / 2$.

Summarizing, we obtain the existence of a bounded sequence $C_{k}^{\prime}$ such that

$$
-\frac{\gamma}{2}\left\|u_{k}\right\|^{2}-\mu_{1}^{k} \nabla g_{1}\left(u_{k}\right) u_{k} \geq o(1)\left\|u_{k}\right\|+C_{k}^{\prime},
$$

and this immediately gives that both $\mu_{1}^{k}$ and $\left\|u_{k}\right\|$ are bounded sequences.

Now let $\left(u, \lambda_{1}, \lambda_{2}\right) \in \Lambda$ such that (up to subsequences) $u_{k} \rightarrow u$ in $H, \lambda_{i}^{k} \rightarrow \lambda_{i}$, $i=1,2$. That $\left\|u_{k}\right\| \rightarrow\|u\|$ now follows by a standard proof, giving that the 
convergence of $u_{k}$ to $u$ is indeed strong in $H$. In fact, by the strong convergence of $u_{k} \rightarrow u$ in $L^{p}$ for all $p<\infty$ ensured by the Sobolev compact embedding, we know that

$$
\lambda_{i}^{k} \nabla G_{1}\left(u_{k}\right)\left(u_{k}-u\right) \rightarrow 0, \quad i=1,2
$$

(since $u_{k}$ is bounded). Moreover (up to subsequences)

$$
\nabla J\left(u_{k}\right)\left(u_{k}-u\right)=\lim _{k \rightarrow \infty}\left(\left\|u_{k}\right\|^{2}\right)-\|u\|^{2}
$$

and thus, testing (23) by $v_{k}=u_{k}-u$ we obtain the desired convergence property.

3.4. End of the proof. Thanks to Lemma 3.8, in order to recover compactness, we can replace $g_{2}$ with

$$
\tilde{g}_{2}(u):=g_{2}(u)-a[J(u)-c],
$$

where $c$ is the minimax level defined in (21) and

$$
a=\frac{1}{2 \max _{u \in \gamma_{0}(\partial Q)}|J(u)-c|} .
$$

Note that $a$ is finite and strictly positive; in fact $\gamma_{0}(\partial Q)$ is compact and $\mid J(\cdot)-$ $c \mid$ is continuous on $H$; moreover since for $z=(1 / \delta, \ldots, 1 / \delta) \in \partial Q$ it holds that $J\left(\gamma_{0}(z)(\cdot)\right)<0$ (if $\delta$ is small enough), then

$$
\max _{u \in \gamma_{0}(\partial Q)}|J(u)-c|>c>0 .
$$

Next we consider the variational setting (related to $\tilde{g}_{2}$ instead of $g_{2}$ ):

$$
\begin{array}{cl}
\widetilde{\Gamma}=\Gamma \quad:=\left\{\gamma(\cdot)=\eta\left(t, \gamma_{0}(\cdot)\right): t \geq 0\right\}, \\
\widetilde{E} & :=\left\{g_{1}<1, \tilde{g}_{2}<1\right\}, \\
\widetilde{T}_{\gamma} & :=\{z \in Q: \gamma(z) \in \bar{E}\}, \\
\widetilde{c} & :=\inf _{\gamma \in \Gamma} \max _{z \in \widetilde{T}_{\gamma}} J(\gamma(z)) .
\end{array}
$$

Note that the theory in Section 2 applies to this structure provided the following basic property holds:

$$
\gamma(\partial Q) \subset\{J<0\} \cup\left\{\tilde{g}_{2}>1\right\} .
$$

In fact this is true thanks to the choice of $a$ : if we take $z \in \partial Q$ such that $g_{2}(\gamma(z))>$ $3 / 2$, then

as desired.

$$
\tilde{g}_{2}(\gamma(z)) \geq g_{2}(\gamma(z))-a \max _{u \in \gamma(\partial Q)}[J(u)-c]>1
$$

Furthermore, by Lemma 3.6 we immediately obtain

Lemma 3.9. $\widetilde{T}_{\gamma} \neq \emptyset$ for all $\gamma \in \Gamma$ and $\tilde{c}>0$.

Proof. Indeed, we know by the quoted lemma that $T_{\gamma} \neq \emptyset$ for all $\gamma \in \Gamma$. That means, $\exists z \in Q$ such that $g_{1}(\gamma(z)) \leq 1, g_{2}(\gamma(z)) \leq 1$ and $J(\gamma(z)) \geq c$. Thus $\tilde{g}_{2}(\gamma(z)) \leq g_{2}(\gamma(z))$ and we conclude that $t \in \widetilde{T}_{\gamma}$, that is, $T_{\gamma} \subset \widetilde{T}_{\gamma}$ for all $\gamma \in \Gamma$. Moreover, the same analysis allows us to conclude that $\tilde{c} \geq c$ and since $c$ is positive, the $c>0$.

Now we are ready to state and prove our main existence result.

Theorem 3.1. There exists $u \in H$ critical point of $J$ (at level c) having exactly $2 n$ zeroes. 
Proof. Let us first remark that $J$ satisfies the $C P S_{c}$ condition relative to the involved constraints; thus by direct application of Corollary 2.1 we obtain $u \in\left\{G_{1}<\right.$ $\left.1, G_{2}<1\right\}$ such that $J(u)=c$ and $\nabla J(u)-\lambda_{1} G_{1}(u)+\lambda_{2} G_{2}(u)=0$ for some $\lambda_{i} \in[0,1]$. But since $G_{1} \equiv g_{1}$ and $G_{2}(u)=g_{2}(u)$, it turns out by Remark 3.1 that $\nabla J(u)=0$. Thus $u$ is a critical point for $J$ and since it indeed belongs to $\left\{g_{1}<1, g_{2}<1\right\}$, it has the required sign properties (recall Remark 3.1 again).

\section{ACKNowledgments}

The authors are indebted to the unknown referees for their sharp remarks and encouraging comments.

\section{REFERENCES}

[1] A.U. Afuwape, J. Mawhin, F. Zanolin, An existence theorem for periodic solutions and applications to some nonlinear ordinary and delay equations, preprint

[2] T. Bartsch, M. Willem, Infinitely many radial solutions of a semilinear elliptic problem on $\mathbb{R}^{N}$, Arch. Rat. Mech. Anal. 124 (1993), 261-276 MR 94g:35078

[3] D. Bonheure, L. Sanchez, M. Tarallo, S. Terracini, Heteroclinics connections between nonconsecutive equilibria of a fourth order differential equation, Calculus of Variation and PDE's, to appear

[4] J. Chaparova, Existence and numerical approximations of periodic solutions of semilinear fourth-order differential equations, J. Math. Anal. Appl. 273 (2002), 121-136 MR 2003j:35041

[5] M. Conti, L. Merizzi, S. Terracini, Radial solutions of superlinear equations on $R^{N}$. I. A global variational approach, Arch. Ration. Mech. Anal. 153 (2000), 291-316 MR 2001j:35053

[6] M. Conti, S. Terracini, Radial solutions of superlinear equations on $R^{N}$. II. The forced case, Arch. Ration. Mech. Anal. 153 (2000), 317-339 MR 2001j:35054

[7] M. Conti, S. Terracini, G. Verzini, Nodal solutions to a class of nonstandard superlinear equations on $R^{N}$, Adv. Differential Equations 7 (2002), 3, 297-318 MR 2003i:35083

[8] F.L. Garcia, Periodic solutions of 4 th order O.D.E., Dynam. Contin. Discrete Impuls. Systems 7 (2000), 239-254 MR 2001a:34070

[9] P. Habets, L. Sanchez, M. Tarallo, S. Terracini, Heteroclinics for a class of fourth order conservative differential equations, Equadiff 10, Prague 2001, CD-ROM Proceedings

[10] A.C. Lazer, P.J. McKenna, Large amplitude oscillations in suspension bridges: some new connections with nonlinear analysis, SIAM Rev. 32 (1990), 537-578 MR 92g:73059

[11] J. Mawhin, F. Zanolin, A continuation approach to fourth order superlinear periodic boundary value problems, Topol. Methods Nonlinear Anal. 2 (1993), 55-74 MR 95a:34063

[12] C. Miranda, Un'osservazione sul teorema di Brouwer, Boll. U.M.I. Serie II, Anno II, 1 (1940), 5-7. MR 3:60b

[13] V.Y. Mizel, L.A. Peletier, W.C. Troy, Periodic phases in second order materials, Arch. Rat. Mech. Anal. 145 (1998), 343-382 MR 99j:73011

[14] Z. Nehari, Characteristic values associated with a class of nonlinear second order differential equations, Acta Math. 105 (1961), 141-175 MR 23:A1097

[15] L.A. Peletier, W.C. Troy, Spatial patterns described by the Fisher-Kolmogorov equation: Kinks, Diff. Int. Eq. 8 (1995), 1279-1304 MR 96c:35182

[16] L.A. Peletier, W.C. Troy, A topological shooting method and the existence of kinks of the extended Fisher-Kolmogorov equation, Topol. Meth. Nonlinear Anal. 6 (1995), 2, 331-355 MR 97d:34031

[17] L.A. Peletier, W.C. Troy, Spatial patterns described by the Fisher-Kolmogorov equation: periodic solutions, SIAM J. Math. Anal. 28 (1997), 1317-1353 MR 98k:35198

[18] L.A. Peletier, W.C. Troy, Multibump periodic traveling waves in suspension bridges, Proc. Roy. Soc. Edimburgh, Sect. A 128 (1998), 3, 631-659 MR 99h:34038

[19] B.R. Rynne, Global bifurcation for $2 m$-th order boundary value problems and infinitely many solutions of superlinear problems, preprint 
[20] D. Smets, J.B. van den Berg, Homoclinic solutions for Swift-Hohenberg and suspension bridge type equations, J. Diff. Eq. 184 (2002), 78-96

[21] M. Struwe, Superlinear elliptic boundary value problems with rotational symmetry, Arch. Math. 39 (1982), 233-240 MR 84a:35097

Dipartimento di Matematica del Politecnico, Piazza Leonardo da Vinci, 32 - 20133 Milano (I), Italy

E-mail address: monica.conti@polimi.it

Dipartimento di Matematica e Applicazioni, Università di Milano Bicocca, Via BicocCA Degli Arcimboldi 8, 20126 Milano (I), Italy

E-mail address: suster@matapp.unimib.it

Dipartimento di Matematica del Politecnico, Piazza Leonardo da Vinci, 32 - 20133 Milano (I), Italy

E-mail address: gianmaria.verzini@polimi.it 